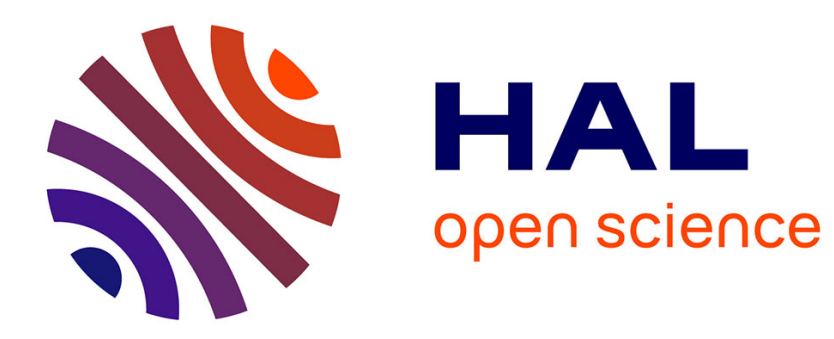

\title{
La Fiction comme Culture de la Simulation
}

Jérôme Pelletier

\section{To cite this version:}

Jérôme Pelletier. La Fiction comme Culture de la Simulation. Poétique : revue de théorie et d'analyse littéraire, 2008, 154, pp.131-146. ijn_00355831

\section{HAL Id: ijn_00355831 \\ https://hal.science/ijn_00355831}

Submitted on 25 Jan 2009

HAL is a multi-disciplinary open access archive for the deposit and dissemination of scientific research documents, whether they are published or not. The documents may come from teaching and research institutions in France or abroad, or from public or private research centers.
L'archive ouverte pluridisciplinaire HAL, est destinée au dépôt et à la diffusion de documents scientifiques de niveau recherche, publiés ou non, émanant des établissements d'enseignement et de recherche français ou étrangers, des laboratoires publics ou privés. 


\title{
«LA FICTION COMME CULTURE DE LA SIMULATION »
}

\author{
POETIQUE (AVRIL 2008, $\mathrm{N}^{\circ}$ 154), PP. 131-146.
}

\author{
Jérôme Pelletier
}

\begin{abstract}
Dans toutes les cultures, les humains passent une grande partie de leur temps à jouer à faire semblant, à penser à des mondes imaginaires, à faire l'expérience des créations imaginaires des autres et toutes les sociétés humaines dont nous avons connaissance possèdent l'art et la fiction. On peut donc penser que la fiction et l'art correspondent à des caractéristiques fondamentales de l'esprit humain et ne sont pas réductibles à de simples phénomènes culturels et historiques (ce qui ne signifie nullement que les valeurs esthétiques elles-mêmes ne soient pas essentiellement historiques). Des recherches en philosophie de l'esprit et en sciences cognitives montrent que le faire semblant et la simulation sont des caractéristiques fondamentales de l'espèce humaine. Dans ce qui suit, je propose une analyse psychologique de diverses formes de simulation activées par la consommation des fictions en faisant dialoguer, dans la mesure du possible, la philosophie de l'esprit, l'esthétique et les sciences cognitives.
\end{abstract}

\section{DE LA « THÉORIE DE L'ILLUSION » À LA «THÉORIE DE LA SIMULATION »}

Quand nous lisons ou voyons une oeuvre de fiction en étant «pris » par le récit, croyonsnous en la réalité des personnages et événements décrits dans ces fictions ? Deux interprétations de cette question doivent être évitées. La question n'est pas de savoir si les fictions peuvent induire des croyances. Les fictions peuvent certainement induire des croyances sur nous-mêmes, sur nos valeurs, sur le monde, sur leurs auteurs et que sais-je encore. La question n'est pas non plus de savoir s'il est possible de se méprendre sur la nature fictionnelle ou non fictionnelle d'un récit. Lorsqu'il y a méprise sur la nature d'un récit fictionnel, on en vient sans doute à tort, à croire en la réalité des personnages et événements représentés dans le récit. La question porte sur la nature de nos interactions psychologiques - dans des conditions standards - avec les fictions. Il s'agit de se demander si les interactions psychologiques standards avec les fictions relèvent d'une manière ou d'une autre de la croyance ? Croit-on, par exemple, en lisant les romans de Doyle, que Sherlock Holmes habite au 221B Baker Street? Croit-on, en lisant le récit de Flaubert, que Emma rencontre Rodolphe à Rouen ? L'explication des émotions que nous sommes susceptibles d'éprouver en lisant ou en voyant une oeuvre de fiction requiert-elle de faire appel aux croyances que l'on aurait sur la réalité des personnages ou événements impliqués dans ces fictions ?

Ces questions sont pertinentes à plus d'un titre. Tout d'abord, le traitement cognitif des fictions requiert - sauf stipulation particulière comme dans la science fiction - la mobilisation de nos croyances sur le monde : s'il pleut le jour de la rencontre d'Emma et de Rodolphe, alors nous en inférons que les rues de Rouen sont mouillées même si le récit de Flaubert ne le précise pas. De même, nous savons que les lois de la physique et de la biologie s'appliquent au monde décrit par Flaubert bien que cela ne soit pas dit explicitement dans le récit. Rien n'interdit qu'un récit de fiction fasse référence - voire ne fasse que référence - à des individus et lieux réels sur lesquels nous 
avons un ensemble de croyances, des croyances dont la mobilisation est alors nécessaire pour comprendre le récit. Enfin et surtout, les oeuvre de fiction ont des effets émotionnels intenses sur leurs lecteurs ou spectateurs. C'est sans doute l'une des raisons pour lesquelles nous chérissons les fictions. Or il semble admis que les émotions présupposent certaines convictions, croyances ou jugements. Si on considère l'émotion de crainte, il semble que l'on ne craigne pour soi-même que si l'on se croit en danger ou bien que l'on ne craigne pour les autres que si l'on croit qu'ils existent réellement et sont en danger.

Ce dernier point sur l'intensité des effets émotionnels des fictions a sans doute motivé des théoriciens de la fiction - en particulier des théoriciens des fictions cinématographiques (Bazin 1985 ; Ellis 1982 ; Balazs 1972) - à soutenir que cette intensité résultait d'un «effet de réalité » produit par le film sur ses spectateurs. Faisant parfois appel à des considérations psychanalytiques (cf. Metz 1977), ces théoriciens en sont venus à défendre une conception «illusionniste » de nos interactions avec les fictions. Selon la «théorie de l'illusion », les lecteurs ou spectateurs de fictions au fait de la nature fictionnelle du récit en viendraient à croire en la réalité des personnages et événements représentés.

Insatisfait par cette «théorie de l'illusion », Currie (1990,1995a) a proposé une conception alternative de nos interactions psychologiques avec les fictions. Suivant en cela Walton (1990), Currie soutient que les fictions sont des accessoires («props ») que nous utilisons dans des jeux de faire semblant. Les fictions engagent non pas les croyances des lecteurs ou des spectateurs mais leurs capacité à faire semblant et à imaginer et constituent autant de guides ou d' " aides externes » pour leurs imaginations (Currie, 1995b, p.151 ; 1995c, p. 254). Selon cette conception alternative, lorsque nous lisons ou voyons une fiction et somme pris par le récit, nous ne croyons pas en sa réalité mais imaginons sa réalité : les fictions ne sont pas sources d'illusions ou de croyances fausses mais des « déclencheurs » d'imagination et de faire semblant. ${ }^{1}$

Cette conception alternative n'a au premier abord rien de révolutionnaire : les premières réflexions sur les œuvres de fiction ont tout naturellement établi une relation entre ces œuvres et l'exercice de l'imagination, que ce soit pour décrire l'activité des auteurs de fictions ou pour décrire l'activité des lecteurs ou spectateurs de fictions. Ce qui est relativement nouveau dans cette conception- et que l'on doit pour partie à Ryle (1949, pp. 245-72) - est la description de l'imagination dans les termes d'un «faire semblant», d'un «faire comme si ». Ce qui est aussi relativement nouveau - et que l'on doit cette fois-ci, dans le cadre d'une réflexion sur l'image, pour partie à Gombrich (1965)- est le rapprochement effectué entre les fictions sophistiquées des adultes (romans, films, ...) et les jeux de faire semblant de l'enfance. Surtout, cette approche de nos interactions avec les fictions en termes de faire semblant permet aux théoriciens de la fiction de bénéficier des découvertes qui ont été faites dans les sciences cognitives aussi bien sur la simulation mentale que sur l'imitation, la mimèsis et les systèmes miroirs. ${ }^{2}$

\section{LA SIMULATION EN PHILOSOPHIE DE L'ESPRIT ET DANS LES NEUROSCIENCES COGNITIVES}

La simulation a attiré l'attention des chercheurs ces dernières années, tout particulièrement l'attention des philosophes à l'intersection de la philosophie et de la psychologie qui se sont intéressés aux bases de la «psychologie naïve ». Les phénomènes de simulation ont également attiré l'attention des chercheurs des neurosciences cognitives, en particulier les chercheurs des neurosciences du système moteur. La découverte par ces derniers des neurones miroirs laisse penser

1 En France, il importe de mentionner Schaeffer (1999) qui montre que la fiction ludique partagée - à la différence de la feintise sérieuse - ne vise pas à tromper ou à induire en erreur. .

Les remarques qui suivent s'inscrivent dans le prolongement de la thèse de Schaeffer (1999) sur l'existence de mécanismes imitatifs ancestraux à la base du processus représentationnel mis en œuvre par la fiction ludique. 
que ceux-ci sont la base neurale des processus de simulation, ce qui inclut les processus décrits par les philosophes de l'esprit. En outre, il semblerait que la compréhension empathique d'autrui reposerait elle aussi sur des processus de simulation et, au moins en partie, sur des systèmes de neurones miroirs. Expliquons ces divers points.

La «psychologie naïve » est, au même titre que la physique naïve, la cognition numérique, la biologie naïve et la géométrie naïve, un des sous-systèmes du système de conceptualisation du monde. La psychologie naïve est aussi appelée «mentalisation» ([«mindreading »]) dans la terminologie de Baron-Cohen (1995) ou encore «théorie de l'esprit ». Ce sont autant de noms de cette capacité cognitive grâce à laquelle les êtres humains perçoivent et conceptualisent les comportements de leurs congénères et s'attribuent les uns aux autres des états mentaux intentions, désirs, croyances, émotions, etc. — qui sont les causes de leurs actions.

Une controverse a eu lieu en philosophie de l'esprit sur les mécanismes mentaux qui rendent possible la mentalisation et la théorie de l'esprit. Cette controverse a opposé les avocats de la «théorie de la théorie » à ceux de la «théorie de la simulation». (Cf. Davies et Stone 1995a, 1995b ; Carruthers et Smith 1996, Heal 1994, Dokic et Proust 2002). Selon la théorie dite de la simulation « off-line » (Gordon 1995a, 1995b, 1996 ; Goldman 1992) nous attribuons à autrui des états mentaux en utilisant notre propre esprit comme un modèle de son esprit, en re-créant, répliquant ou imitant dans notre esprit les états mentaux -croyances, désirs, intentions, perceptions...- d'autrui. Comme les états mentaux que nous re-créons dans notre esprit pour imiter la vie mentale d'autrui ne nous mènent pas à agir réellement sur la base de ces états puisqu'ils sont destinés à être attribués à autrui, on parlera de simulation «off-line » au sens où ces états simulés ne donnent pas lieu à l'action.

Kahneman \& Tversky (1979) voyaient la simulation mentale comme une méthode pour prédire les émotions d'autrui. Dans un test devenu célèbre, ils donnèrent à des sujets un scénario dans lequel deux individus $\mathrm{A}$ et $\mathrm{B}$ se rendent au même aéroport pour prendre deux avions différents décollant à la même heure. A et $\mathrm{B}$ ratent tous les deux leur avions. Mais à la différence de ce qui s'est passé pour $\mathrm{A}$, le scénario stipule que l'avion de $\mathrm{B}$ a été retardé d'une manière telle que $\mathrm{B}$ ne rate son avion que de quelques minutes. La question alors posée aux sujets est la suivante : qui de $\mathrm{A}$ ou B est le plus peiné d'avoir raté son avion ? 96\% des sujets répondent que B est plus peiné que A d'avoir raté son avion. Selon Kahneman \& Tversky, les sujets soumis au test prédisent les états mentaux de $\mathrm{A}$ et $\mathrm{B}$ en simulant ou s'imaginant à la place de $\mathrm{A}$ ou de $\mathrm{B}$ et en comparant leurs expériences.

Selon certains philosophes comme Gordon (1995a, 113), la capacité cognitive de simulation - à la base de la mentalisation - reposerait sur des mécanismes imitatifs superficiels, des mécanismes cognitifs nous permettant de reproduire en nous des états se trouvant dans d'autres individus ou organismes. Ces mécanismes imitatifs seraient notamment à la base des phénomènes de «contagion émotionnelle » (Scheler, 1954). Pour Gallese et Goldman (1998) également, une partie significative des épisodes de mentalisation reposerait sur des processus superficiels d'imitation. Si l'on suit cette interprétation philosophique, la simulation mentale serait donc une des formes de l'imitation.

Les sciences cognitives se sont, dans un mouvement parallèle à celui des philosophes de l'esprit, également intéressé à un certain nombre de mécanismes d'imitation. Ainsi, Meltzoff et Moore (1993) ont analysé les imitations par les bébés humains des mimiques du visage. Ils ont constaté que des bébés humains en vie depuis 42 minutes sont capables d'imiter des mouvements bucco-faciaux, comme l'ouverture de la bouche et la protrusion de la langue. Surtout, la célèbre découverte par Rizzolatti et al. (1988) de l'existence dans le cortex pré-moteur du singe macaque dans l'aire F5 de neurones visuomoteurs dits "miroirs" a constitué une avancée considérable dans l'étude des phénomènes d'imitation. Ces neurones sont dits miroirs parce qu'ils déchargent non seulement lorsque l'animal effectue certaines actions manuelles dirigées vers des objets mais aussi lorsqu'il perçoit les mêmes actions effectuées par un autre animal ${ }^{3}$. Comme l'observation par le 
singe macaque de mouvements particuliers des mains vers un certain type d'objets activent automatiquement dans son cerveau une réplique du plan d'action dont il observe l'exécution, les neurones miroirs constitueraient la base neurale chez les primates d'un système d'imitation.

Ces neurones miroirs ont ensuite été découverts chez l'homme (Fadiga et al., 1996) dans le cerveau duquel ils sont plus disséminés que dans le cerveau du singe macaque et constituent un véritable système de neurones miroirs. Chez l'homme, des études en imagerie cérébrale ont montré que les zones cérébrales activées dans la préparation, la simulation et l'observation d'une action se superposent en partie avec celles qui s'activent lors de la génération d'une action intentionnelle (Decety \& Grèze,1999; Chaminade et al., 2002). Dans le même sens, Decety, Jeannerod \& Prablanc (1989) ont montré que la durée d'une action que l'on simule mentalement est la même que celle qui est requise pour exécuter l'action. De même, une étude de Decety \& al.(1997) a montré que l'observation d'autrui active la représentation dynamique de l'action correspondante exécutée par l'agent. Les zones cérébrales ainsi activées (STS (sillon temporal supérieur), LPS (lobule pariétal supérieur, Ba7) et PM (aire prémotrice dite aire de Broca)) formeraient un système qui, en quelque sorte, apparie l'observation et l'exécution d'une action via le déroulement mental - c'est-àdire la simulation mentale - de la représentation du plan (en termes de moyens et de fins) de l'action observée. Comme ces neurones miroirs déchargent aussi bien pendant la planification de ses propres actions que pendant l'observation des mêmes actions chez autrui, on peut faire l'hypothèse, en suivant Goldman (2000) que le système des neurones miroirs constituerait l'architecture neurale des phénomènes d'imitation et de «résonance » mentale, l'architecture neurale de la simulation dont, comme on l'a vu, certains philosophes de l'esprit pensent qu'elle est à la base de notre compréhension d'autrui.

Enfin, des recherches (Wicker et al. 2003) ont été menées sur les relations entre l'expérience «en première personne» d'émotions fortes comme le dégoût et l'observation «en troisième personne » de cette même émotion telle qu'elle peut être exprimée par la mimique faciale d'un sujet. Il apparaît que la même structure neurale miroir au niveau de l'insula antérieure est activée par les deux expériences mentionnées. Cela suggère - au moins pour les émotions comme celles du dégoût - que les expériences en première et en troisième personne de ces émotions partagent le même sous-bassement neural. Les phénomènes d'empathie qui nous conduisent, à partir de la perception en troisième personne de l'expression par exemple faciale d'un état affectif, à faire l'expérience en première personne d'une version approchante du même état affectif et, de cette manière, à comprendre l'état affectif d'autrui reposeraient également, au moins en partie, sur des systèmes miroirs. Un mécanisme de reconnaissance des émotions basé sur la simulation par « résonance sans médiation »a été proposé par Goldman et Sripada (2004). Decety et Jackson (2004) développent une approche analogue. Pour décrire ces phénomènes, Gallese (2005) parle de « simulation incarnée » [« embodied simulation»], référant par là à un mécanisme fonctionnel de notre cerveau susceptible de nous donner une expérience directe de la vie mentale d'autrui. Il apparaît, selon ces chercheurs, que les mécanismes miroirs, originairement découverts dans le système moteur, pourraient constituer une structure organisationnelle fondamentale de notre cerveau.

Il ressort de ces descriptions de la simulation en philosophie de l'esprit comme de ces recherches dans les neurosciences cognitives que la capacité de simulation serait une capacité générale ou polyvalente - c'est-à-dire une capacité non limitée à un domaine particulier - à utiliser un système cognitif «off-line» - qu'il soit visuel, auditif, émotionnel, moteur, inférentiel, décisionnel ... avec des entrées et/ou des sorties qui sont/peuvent être des états simulés. ${ }^{4}$ Quelle est

$4 \quad$ Cette hypothèse d'une capacité de simulation "massive" dont le domaine recouvrerait la diversité des systèmes mentionnés a suscité de nombreuses discussions critiques dont un colloque virtuel s'est fait, en 2004/2005, l'écho (cf. "What do mirror neurons mean?" à http://www.interdisciplines.org/mirror/). Pour une étude des relations entre les phénomènes de contagion motrice et de contagion émotionnelle, voir Grèzes \& de Gelder (2005); Magnée, Stekelenburg, Kemner \& de Gelder, B. (2007). 
la fonction de cette capacité ? Il semble que les diverses descriptions des mécanismes de simulation qui viennent d'être données favorisent l'hypothèse que ces mécanismes viseraient, de manière diverse, à donner du sens aux comportements d'autrui en adoptant sa perspective. Selon cette interprétation, les mécanismes de simulation auraient d'abord une fonction pour la cognition sociale (Humphreys 1983) ${ }^{5}$. Qu'en est-il des mécanismes de simulation dans la cognition des fictions ?

Les mondes imaginaires établis par les fictions sont les objets de nos attitudes psychologiques sans qu'il soit possible d'interagir physiquement avec eux. On peut faire l'hypothèse que l'impossibilité d'agir sur la fiction - et la nécessaire inhibition des réactions motrices consécutive de cette impossibilité - permet aux consommateurs de fictions de consacrer une grande partie de leurs ressources cognitives aux tâches de simulation. En second lieu, il semble que les créateurs d'œuvres d'art - en particulier dans les arts visuels et la musique - sont conduits à résoudre certains problèmes représentationnels propres à leur art en s'appuyant sur la capacité de simulation des destinataires de leurs ouvres. La fiction peut alors être comprise, suivant cette double hypothèse, comme une véritable «culture de la simulation », une exploitation, parfois très raffinée et complexe, à des fins ludiques ou artistiques de capacités de simulation plus ou moins primitives. Dans ce qui suit, nous présentons tout d'abord la typologie des simulations mobilisées par les fictions narratives telle qu'elle a pu être esquissée par Currie. Il apparaitra que ces simulations sont toutes cognitivement "de haut niveau" puisqu'il s'agit de simulations souvent indirectes de croyances, de désirs, ou d'être dans la situation de tel ou tel être réel ou imaginaire. Dans la dernière partie de l'article, nous faisons l'hypothèse que dans les arts visuels et musicaux une hypothèse peut-être également pertinente pour la poésie et la littérature - , des formes cognitivement moins élevées et plus directes de simulation - les simulations "par résonance" - sont sollicitées par les artistes, des formes de simulation dont la mise en œuvre expliquerait une partie de l'expressivité des oeuvres.

\section{SIMULATIONS « IMPERSONNELLES » ET « PERSONNELLES » DANS LA FICTION NARRATIVE}

Currie $(1990,1995 a)^{6}$ a souligné le rôle de la simulation mentale dans nos interactions avec les fictions. Il est vrai que les objections adressées à la théorie de la simulation «off-line » en tant que théorie de la compréhension des états mentaux d'autrui hors fiction ${ }^{7}-$ la simulation ne nous permettrait pas d'avoir une connaissance des états mentaux d'autrui ou elle ne nous donnerait pas, en fait, une telle connaissance - ne constituent pas nécessairement des objections à la théorie de la simulation «off-line » comme théorie de nos interactions psychologiques avec les fictions. Selon Currie, le lecteur ou le spectateur répond aux fictions en adoptant des états mentaux qui sont des simulations ou des répliques «off-line», des répliques notamment d'états représentationnels comme les croyances et les désirs. Les croyances et désirs suscitées par les fictions sont des simulations, pour Currie, au sens où ces états ont le même contenu que les croyances et désirs réels, les mêmes rôles inférentiels, les mêmes pouvoirs affectifs que leurs répliques réelles ${ }^{8}$, mais ces états

5 Currie (1995c) défend l'hypothèse que la capacité de simulation a pour fonction de permettre la planification de stratégies d'action.

$6 \quad$ Voir également Walton (1997a) et Feagin (1996), deux philosophes qui établissent d'étroites connexions entre leurs théories sur la fiction et la théorie de la simulation mentale.

$7 \quad$ Cf. les débats au colloque virtuel mentionné plus haut ( "What do mirror neurons mean?" à http://www.interdisciplines.org/mirror/). Voir également Heal (1994: 136). Pour une critique de la généralisation des mécanismes de résonance motrice à la cognition sociale, cf. Jacob (2002), Jacob \& Jeannerod (2005).

8 Currie, après avoir soutenu que les émotions de la fiction étaient des «quasi-émotions » (Currie 1990, p. 183sq.), a récemment soutenu que l'on a des émotions réelles devant ce qui est simplement simulé ou imaginé (Currie et Ravenscroft, 2002, p. 196). La thèse que la simulation ne 
ne sont pas connectés au comportement et à l'action comme le sont les croyances et les désirs réels. L'effet de la fiction n'est donc pas de créer une illusion de réalité et des croyances fausses sur les situations décrites dans la fiction. La fiction fait son effet en nous engageant à avoir des états mentaux qui sont des répliques de croyances et de désirs, voire des répliques d'émotions réelles.

Entrons dans le détail. Currie distingue trois grands types de simulations dite «personnelles» déclenchées par les fictions narratives: la simulation d'être dans la position de quelqu'un apprenant les faits décrits dans le récit à partir d'une source fiable, la simulation des états mentaux des personnages décrits dans le récit, et la simulation d'être dans la position de l'auteur (inféré) du récit. Ces trois exercices de la simulation suscités par les œuvres de fiction relèvent de la simulation «personnelle» au sens où, dans ces simulations, le sujet s'implique lui-même: il s'imagine lui-même être l'auteur (inféré), il s'imagine lui-même être quelqu'un qui a une relation épistémique fiable avec la source du récit ou il s'imagine lui-même dans la situation de tel ou tel personnage. Par contraste, Currie insiste sur le fait que lorsque le lecteur ou le spectateur imagine le contenu fictionnel de l'histoire, il imagine alors simplement que ceci et cela ont lieu sans imaginer avoir un quelconque contact épistémique «personnel» avec ce qui a lieu, ou sans simuler la situation de tel ou tel personnage ou «auteur inféré ». L'imagination du contenu fictionnel de l'histoire est, pour Currie, un exercice de la simulation « non personnelle ${ }^{9}$.

A la différence des simulations non personnelles, les simulations de type personnel - et ici se joue une grande partie du débat entre Walton (1990) et Currie (1990) ${ }^{10}$ - ne sont pour Currie que secondaires, instrumentales et optionnelles. Seules les simulations impersonnelles et propositionnelles caractérisent de manière fondamentale, selon Currie, nos interactions avec les fictions. Quand je lis ou je vois Peter Pan, j'ai des simulations de croyances ayant pour contenus: que Peter est en danger, que Capitaine Crochet a peur du crocodile.... Le récit de Peter Pan me conduit également à avoir des simulations de désirs ayant pour contenu, par exemple, que Peter échappe à Crochet... Dans aucune de ces simulations impersonnelles, soutient Currie, le lecteur ou le spectateur de Peter Pan ne s'imagine dans l'esprit d'un personnage : simuler la croyance que Peter est en danger ne requiert pas de simuler les croyances de Peter (il se peut que Peter ignore le danger qui le menace). L'exercice de la simulation impersonnelle consiste à insérer tel ou tel contenu propositionnel du récit dans des mécanismes psychologiques appropriés (croyances, désirs) fonctionnant « off-line ».

Il reste que Currie admet que le lecteur ou le spectateur peut être conduit à simuler la situation de tel ou tel personnage. «Ce qui est si souvent appelé l'identification du public avec un personnage », soutient Currie, "est mieux décrit en termes de simulation mentale de la situation d'un personnage par le public » (Currie, 1995c, p. 257). Mais la simulation des états mentaux d'un personnage de fiction ne se fait pas de la manière dont nous simulons les états mentaux d'autrui hors de la fiction. Dans la fiction, il ne suffit pas de se mettre à la place des personnages pour les simuler. Lorsqu'il s'agit de fiction littéraire ${ }^{11}$, la simulation de la vie mentale d'un personnage d'une fiction littéraire requiert, de la part du lecteur, qu'il simule la position d'un lecteur d'un compte-rendu factuel, qu'il se mette à la place de ce lecteur imaginaire et simule ses états mentaux. Il s'agit pour le lecteur de s'imaginer lui-même - un cas de simulation «personnelle » - « dans la

donne pas lieu à d'authentiques émotions a constitué le principal motif d'opposition de Carroll (1990) à la thèse que la théorie de la simulation expliquerait nos relations aux fictions.

9 Ce que j'appelle «simulation » personnelle et impersonnelle est décrit par Currie en termes d' « imagination » personnelle et impersonnelle. Sur la distinction de l'imagination «personnelle » et «non personnelle », cf. Currie, (1995a), pp. 164-197.

10 Voir en particulier les réponses de Walton (1997b) aux objections que lui adresse Currie (1995a, pp. 170-9).

11 L'un des leitmotivs de Currie (1995a) est la réduction de la différence - du point de vue psychologique - entre les narrations littéraires et les narrations visuelles, (cf. Currie 1995a, pp. 1967). On peut donc penser que ce qui suit concernant la simulation d'un personnage dans une fiction littéraire vaut -mutatis mutandis - également pour les fictions cinématographiques. 
peau » de quelqu'un qui lirait un récit factuel, voire «dans la peau » de quelqu'un qui aurait certaines propriétés différentes des siennes, des propriétés qui seraient en partie celles du narrateur - lorsque le récit possède un narrateur explicite - et en partie celles de l'auteur du récit. Ce lecteur imaginaire d'un récit factuel est alors supposé, pour attribuer et connaître les états mentaux des personnages du récit, simuler à son tour, de manière personnelle, la vie mentale des personnages dont il lit les aventures. Il se met à avoir des croyances sur la vie mentale des personnages, des désirs pour eux et des sentiments à leur égard sur la base de ses propres simulations. Nous, lecteurs d'un récit fictionnel, afin de simuler la vie mentale des personnages du récit, devons, en quelque sorte, doublement simuler de manière personnelle ou superposer deux niveaux de simulation puisque nous devons simuler quelqu'un qui simule les personnages du récit. Selon le modèle proposé par Currie, ce que l'on appelle communément l'identification d'un lecteur avec un personnage correspond à la simulation, par ce lecteur, des simulations de ce personnage par quelqu'un d'imaginaire qui lirait le récit de fiction comme un récit factuel ${ }^{12}$.

Enfin, si le but est d'interpréter un récit de fiction, de saisir l'histoire narrée par un texte ou un ensemble d'images (éventuellement sonores), un troisième type de simulation personnelle est requis, selon Currie, de la part du lecteur ou du spectateur : la simulation d'être dans la position de l'auteur (inféré) du récit. A partir de l'observation qu'un texte ou un film de fiction est le résultat d'un certain comportement intentionnel, Currie fait l'hypothèse que la saisie de l'histoire narrée par le texte ou le film requiert, à rebours, d'expliquer le comportement intentionnel qui en est la cause. Currie voit une analogie stricte entre l'interprétation d'une œuvre de fiction et l'interprétation du comportement d'un individu. De la même manière que l'interprétation du comportement suppose au moins dans une certaine conception - de formuler des hypothèses sur les états mentaux qui ont causé ce comportement, l'interprétation d'une œuvre suppose de formuler des hypothèses sur ses causes intentionnelles. Dès lors, donner du sens à un récit -saisir l'histoire narrée - suppose de détecter et d'attribuer à son auteur (inféré) ${ }^{13}$ les états mentaux qui sont la cause du récit de la même manière que l'explication du comportement d'un agent requiert de lui attribuer croyances, désirs et intentions. ${ }^{14}$ Comme les états mentaux qui sont la cause du récit sont des intentions narratives particulières, l'interprétation d'un récit de fiction revient, selon Currie, à formuler des hypothèses sur les intentions narratives de son auteur (inféré). Et le contenu des intentions narratives de l'auteur (inféré) est le contenu de l'histoire narrée. En tant que théoricien de la simulation mentale, Currie voit naturellement dans la simulation «off-line» la mécanisme cognitif de connaissance des intentions narratives de l'auteur (inféré) :

«Je me place dans la position de l'auteur, pensant à moi-même comme la personne ayant produit le texte et je me demande «Quelles intentions narratives auraient pu me conduire à écrire ce texte ? » Si je peux faire tourner une simulation dans laquelle le fait d'avoir ces intentions narratives conduit à la décision («off-line») d'écrire ce texte, alors je peux conclure qu'il est au moins plausible que l'auteur a eu ces intentions (Currie, 1995a, 242). » 15

12 Sur tout ceci, cf. Currie (1997, pp. 67-9).

13 L' «auteur inféré » d'une œuvre ne correspond pas nécessairement à son auteur réel, et encore moins, lorsque figure dans l'œuvre un narrateur explicite, à ce narrateur. Pour Currie, l'auteur inféré est l'agent responsable du récit en tant que celui-ci est un récit de fiction alors que son éventuel narrateur explicite est supposé raconter ce qui est de la fiction comme s'il en avait une connaissance réelle. Cf. par exemple, Currie (1995d, p. 20). Pour une défense du concept d' «auteur inféré » d'un film, voir Currie, (1995a, pp. 243-80).

14 Sur tout ceci, cf. Currie (1995a, pp. 235-242).

15 Voir Pelletier (2005) pour une critique de cette conception de l'interprétation des récits de fiction. 


\section{LES SIMULATIONS PAR « RÉSONANCE » DANS LES FICTIONS VISUELLES ET LA MUSIQUE}

Pour Currie, la simulation dans nos interactions avec les fictions est soit impersonnelle et revient à avoir un certain type d'états mentaux - des répliques simulatives d'états représentationnels - soit personnelle et a pour cible des êtres réels ou imaginaires puisqu'il s'agit soit de simuler les états mentaux de quelqu'un qui appréhenderait la fiction comme un compte-rendu factuel, soit de simuler les états mentaux des personnages de fiction (via la simulation précédente), soit de simuler les états mentaux de l'auteur (inféré) du texte ou du film. Cependant, hors fiction, les phénomènes simulatoires n'ont pas toujours une structure aussi complexe et indirecte. Quand un bébé qui n'a que quelques heures de vie imite le sourire d'un adulte, il ne simule pas la situation de l'adulte au sens où il s'imaginerait «dans la peau » de l'adulte afin de saisir l'effet que cela fait de voir le monde d'une manière souriante. Le bébé saisit directement un état émotionnel de l'adulte sur la base de son aptitude pratique à s'engager dans certaines actions d'imitation et de réplication. Une grande partie des états mentaux que l'on attribue aux autres dépend de ce que Goldman et Sripada (2004) appellent la «résonance directe» [ «unmediated resonance »] et qui correspond à des mécanimes de résonance que les actions des autres déclenchent en nous, des mécanismes dont Gallese (2005) considère qu'ils relèvent de la simulation incarnée [«embodied simulation »] et qu'ils décrits comme automatiques, obligatoires, pré réflexifs et inconscients. Les phénomènes d'empathie, de «contagion » ou d' «infection émotionnelle ${ }^{16}$ relèvent de la résonance mentale, de même que notre tendance universelle à mimiquer de manière plus ou moins automatique le comportement expressif d'autres personnes, leurs expressions faciales, leurs gestes et attitudes corporelles, autant de phénomènes d'imitation qui jouent un rôle dans notre compréhension d'autrui. ${ }^{17}$

Comme ces mécanismes fonctionnels de résonance peuvent engendrer hors fiction des contenus représentationnels, on peut faire l'hypothèse que les artistes exploitent dans leur œuvres certains de ces mécanismes. Se plaçant du côté du consommateur des œuvres d'art, Lipps (1903) utilisait déjà le terme d'empathie [«Einfühlung»] pour décrie la psychologie de l'expérience esthétique. Aujourd'hui, dans les sciences cognitives comme en philosophie de l'art, diverses théories sont esquissées mettant en relation nos capacités de résonance -même si le terme « résonance » n'est pas employé - et le pouvoir représentationnel des œuvres d'art. Ainsi, Plantinga (1999) comprend l'utilisation par les cinéastes de gros plans sur les expressions faciales des acteurs comme visant à induire des effets de mimicry chez les spectateurs leur permettant, en retour, de saisir les émotions des personnages. Dans le domaine de la musique, Godoy (2001) défend la thèse que la manière dont on perçoit les sons musicaux dépend étroitement de la simulation automatique - dans l'esprit de l'auditeur - des gestes corporels associés à la production des sons, tels que frapper, souffler, chanter ou des gestes accompagnant la production des sons tels que marcher ou danser... Janata (2001) étaie l'hypothèse de Godoy en montrant que l'imagination des sons active des aires motrices ou pré-motrices du cerveau qui sont également activées lors de l'exécution de gestes corporels. Les découvertes empiriques de ce type montrent que le couplage action/perception et les systèmes de neurones miroirs à la base de certains phénomènes d'empathie seraient également à la base de l'expressivité musicale puisque la perception de cette expressivité résulterait de l'imitation interne des actions et gestes menant à l'expression. Enfin, le philosophe Jerrold Levinson (2002) soutient, à sa manière, que la simulation est à la base de l'expressivité musicale dans le sens où l'écoute de la musique conduirait, selon lui, à simuler la gestuelle expressive d'un agent dans la musique $^{18}$.

$16 \quad$ Cf. Scheler (1954), pp. 14-18.

17 Voir sur ce point les études critiques de Grèzes \& de Gelder (2005) et de Magnée, Stekelenburg, Kemner \& de Gelder, B. (2007).

18 Walton exprime quelques réserves à l'idée que la «mimicry » motrice serait à la base de la perception de l'expressivité musicale (cf. Walton 1999, p. 434). 
Peut-on envisager une explication analogue de l'expressivité de certaines représentations picturales? L'expressivité de certains tableaux résulte-t-elle de l'activation de mécanismes psychologiques de simulation par résonance dans l'esprit du spectateur? Walton (1999) prend l'exemple de l'auto-portrait suivant de Van Gogh (Saint-Rémi, septembre 1889, Musée d'Orsay)

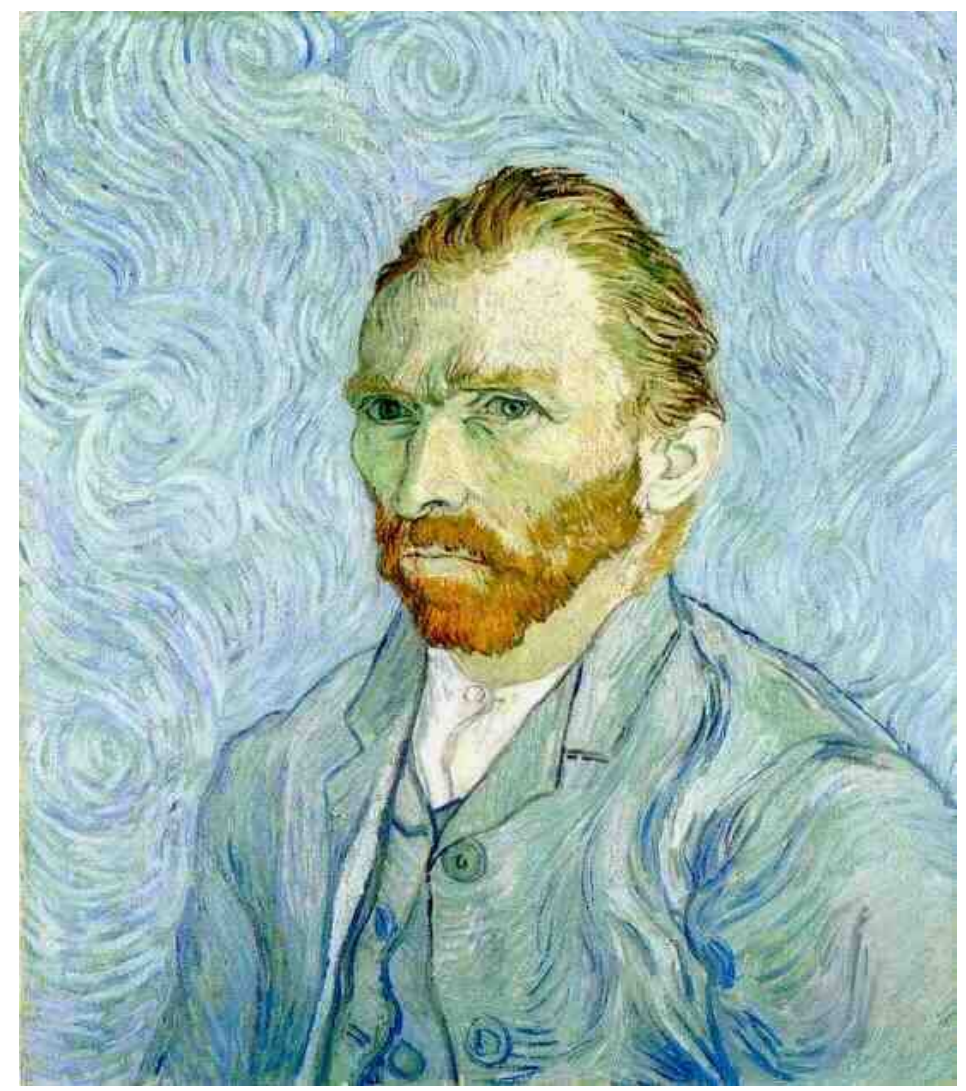

Walton soutient que le tableau «infecte émotionnellement » son spectateur d'une impression de nervosité. A la réflexion, ajoute Walton, ce n'est pas un aspect de l'expression faciale du modèle qui explique cette nervosité mais plutôt des caractéristiques de la peinture elle-même. Plus particulièrement, les coups de pinceau mouvementés à l'arrière plan et sur la veste de Vincent ainsi que sur son visage et sa barbe. Des caractéristiques de l'œuvre, considérées de manière non représentationnelle, expliqueraient, selon Walton, l' «infection émotionnelle » de son spectateur, son sentiment de malaise et de nervosité. Pour Walton, le spectateur de la toile éprouvant une certaine expérience de malaise et de nervosité pense à sa réponse affective nerveuse comme si elle était une réponse empathique à Vincent ${ }^{19}$. Il en est avec la toile de Van Gogh, selon Walton, comme avec la musique de film qui affecte la manière dont on comprend les personnages sur l'écran et les événements qui s'y déroulent. La musique éveille certains sentiments ou émotions dans l'esprit du spectateur qui, s'imaginant alors avoir été infecté par les personnages du film, leur attribue, en retour, ces sentiments et émotions. De la même manière que les spectateurs du film pensent à leur réponse affective à la musique comme étant une réponse empathique aux personnages du film, le spectateur de l'autoportrait de Van Gogh penserait, selon Walton, à sa réponse affective aux coups de pinceau à l'arrière-plan de la toile comme à une réponse empathique à Vincent, la personne représentée dans la toile. ${ }^{20}$

Y a-t-il ici des phénomènes de simulations par résonance qui seraient à la base de cette

$19 \quad$ Cf. Walton (1999: 431).

20 On pourrait envisager un type d'explication analogue d'une partie de l'expressivité de la poésie et des textes littéraires, les coups de pinceau cédant alors la place, dans l'explication, au rythme du vers ou de la phrase, à la prosodie ou à d'autres paramètres non immédiatement représentationnels de la production des œuvres en question. 
« infection émotionnelle »? La perception de la peinture et des coups de pinceau activerait-elle des processus de «mimicry»? Peut-on faire l'hypothèse, en suivant ceux qui soutiennent que l'imagination des gestes corporels à l'origine causale des sons entendus serait au cœur de l'expressivité musicale, que le sentiment de nervosité du spectateur de la toile résulterait de la simulation des gestes corporels «nerveux » qui auraient produits les coups de pinceau sur la toile ? Ce sont autant de pistes que Walton n'évoque pas mais qui mériteraient d'être explorées. Il incombe aux neuroscientifiques, philosophes et théoriciens de l'art d'engager une collaboration pour comprendre le rôle des simulations par résonance dans le pouvoir représentationnel et expressif des images.

\section{CONCLUSION}

De même que nous mettons du sens dans le comportement des autres grâce à la simulation mentale, on peut faire l'hypothèse que la simulation -sous ses diverses formes - est à la base d'au moins une partie du sens que nous trouvons dans les fictions et les œuvres d'art. A suivre cette hypothèse, l'activité culturelle de la fiction apparait, relativement à la cognition sociale qui semble être la fonction propre de la simulation mentale, comme ce que les biologistes évolutionnaires appellent une $«$ exaptation $»^{21}$.

Cette dernière remarque est l'occasion d'une relecture des thèses célèbres de la théoricienne de la littérature, Käte Hamburger (1957) ${ }^{22}$, sur l'importance des verbes mentaux (croire, penser, sentir...) et de ce que l'on a appelé, à la suite de Genette (1972 : pp. 206-224), la «focalisation interne » dans la fiction. Selon Hamburger, le romancier, en quelque sorte, observe l'inobservable, à savoir ce qui se passe dans l'esprit des personnages. Les verbes décrivant les processus intérieurs des personnages comme «penser», «croire», «se souvenir ».. correspondraient, selon Hamburger, à un authentique besoin du romancier. Sitôt que l'on a une occurrence d'une formule comme «Mais, se dit-il, ... », on est, selon Hamburger, dans la fiction. De même, une occurrence de la formule «Vite décidé » dans la phrase «Vite décidé, il se mit à écrire... » fournit la preuve que l'on est dans la fiction: c'est la description d'un processus intérieur -la vitesse d'une décision(à la différence du processus rapporté par «Vite, il sortit son portefeuille »), un processus intérieur, c'est-à-dire, pour Hamburger, un processus inaccessible à l'observation . Hamburger ajoute que le personnage de roman n'est pas un objet que l'on observe mais un sujet. Dans la fiction, la subjectivité d'une tierce personne peut être présentée comme telle. "Elle se souvenait à cet instant des paroles qui lui avaient été dites » est, pour Hamburger, une phrase immédiatement fictionnelle.

Devant ce type de remarques, on peut être conduit à réagir de plusieurs manières. Ou bien on peut faire observer à Hamburger et à ses disciples que ce type de théorie repose sur une conception devenue quelque peu mythologique de la connaissance d'autrui hors fiction, une conception en décalage avec les recherches actuelles sur nos capacités à détecter et identifier hors fiction les états mentaux de nos congénères. La thèse de Hamburger sur l'inaccessibilité de l'intériorité d'autrui à l'observation - une thèse qui est elle-même à la base de sa thèse sur la transparence de l'intériorité des personnages aux auteurs et lecteurs de fiction - semble aujourd'hui battue en brèche par les découvertes sur les systèmes de neurones miroirs et nos diverses capacités de «simulation incarnée », autant de mécanismes qui nous permettent de décoder les émotions, intentions et autres états mentaux d'autrui (hors fiction) et dont la découverte nous incite à ne pas introduire de frontières opaques illusoires entre autrui et nous-mêmes.

$\mathrm{Ou}$ bien on peut soutenir, cette fois-ci, en suivant Hamburger, que comme les fictions littéraires -en particulier, le roman du XIXème siècle- regorgent de descriptions détaillées des états mentaux des personnages, il n'y a certes pas lieu pour le lecteur de simuler quoi que ce soit pour

21 Le plumage des oiseaux a d'abord eu pour fonction de réguler thermiquement le corps avant d'être "exapté" pour permettre le vol. Sur la notion d'exaptation, cf. Gould et Lewontin (1979).

22 Voir également Cohn (1981, 1999). 
savoir ce qui se passe dans l'esprit des personnages - à la différence de ce qui se passe hors fiction puisque le romancier lui dit tout ce qui s'y passe. Mais cette réponse n'exclut pas que les états représentationnels du lecteur d'un roman du XIXème siècle relèvent de la «simulation impersonnelle» au sens de Currie ou d'autres formes de «simulation personnelle » ou de simulations «par résonance ». Il reste qu'il devient difficile de soutenir avec Hamburger, que la narration des pensées internes des personnages en tant que sujets indiquerait la fictionnalité d'une narration $^{23}$. Ces passages narratifs sont en continuité - de par leur contenu - avec des processus de simulation qui ont cours, avant tout, hors fiction. On peut même faire l'hypothèse que les romans du XIXème siècle, en développant une véritable «culture de la simulation » dans l'esprit de leurs lecteurs, loin de manifester un contraste entre le domaine de la fiction et celui de la réalité, aident leurs lecteurs à mieux lire l'intériorité de l'esprit de leurs congénères dans la réalité et à trouver du sens dans leurs comportements. ${ }^{24}$

\section{BIBLIOGRAPHIE}

BALAzS, B.1972 Theory of Film: Character and Growth of a New Art, New York : Arno.

BARon-CoHen, S. 1995 Mindblindness, Cambridge, Mass.: MIT Press.

BAZIN, A. 1985 Qu'est-ce que le Cinéma? Paris : Les Editions du Cerf.

CARroll, N. 1990 The Philosophy of Horror New York Routledge?

CARruthers, P. \& SMith, P. K. (eds.) 1996 Theories of Theories of Mind, Cambridge: Cambridge University Press.

Chaminade, T., Meltzoff, A. N. et Decety, J. 2002 « Does the End Justify the Means ? A PET Exploration of the Mechanisms Involved in Human Imitation », NeuroImage 15, 318-328.

CoHN, D. 1981. La Transparence Intérieure. Paris : Seuil.

CoHn, D. 1999. The Distinction of Fiction. Londres : John Hopkins University Press.

CurRIE, G. 1990. The Nature of Fiction. Cambridge University Press.

CURrIE, G. 1995a. Image and Mind - Film, Philosophy and Cognitive Science Cambridge : Cambridge University Press

CURRIE, G. 1995b. «Imagination and Simulation: Aesthetics meets cognitive science », in A. StOne and M. DAVIES (eds.) Mental simulation: Evaluations and applications, Oxford: Basil Blackwell, 151-169.

23 Sur ce point, voir Pavel (2000: p. 536).

24 Je remercie Y. Citton et J-M Schaeffer pour leurs encouragements. 
CURRIE, G. 1995c. «The Moral Psychology of Fiction », Australasian Journal of Philosophy, Vol. 73, N², 250-259.

CURRIE, G. 1995d. «Unreliability Refigured: Narrative in Literrature and Film », The Journal of Aesthetics and Art Criticism, 53:1, 19-29.

CURrie, G. 1997 : « The Paradox of Caring and the Philosophy of Mind », in M. HJORT \& S. LAVER (eds.), Emotion and the Arts, 63-77.

CurRIE, G. \& RAVEnscroft, I., 2002, Recreative Minds, Oxford: Clarendon Press.

DaVIES, M. \& Stone, T. (eds.), 1995a, Folk Psychology, Oxford: Blackwell.

DAVIES, M. \& Stone, T. (eds.), 1995b, Mental Simulation, Oxford: Blackwell.

Decety, J., Grezes, J., Costes, N., Perani, D., Jeannerod, M., Procyk, E., Grassi, F. \& FAZIO, F., 1997, « Brain Activity During Observation of Action. Influence of Action Content and Subject's Strategy », Brain, 120, 1763-1777.

Decety, J., Jeannerod, M. \& Prablanc, C., 1989, «The Timing of Mentally Represented Actions », Behavioral Brain Research, 34, 35-

DeCETy, J. \& GREZES, J. 1999 NeURAl, « Mechanisms subserving the perception of human actions », Trends Cognitive Science, 3,172-178.

DECETY, J et P. JACKSON 2004, « The Functional Architecture of Human Empathy », Behavioral and Cognitive Neuroscience Reviews, Volume 3, ํ2, 71-100.

Dokic, J. \& Proust, J. (eds.), 2002, Simulation and Knowledge of Action, Amsterdam/Philadelphia: John Benjamins.

Ellis, J. 1982, Visible Fictions: Cinema, Television, Video Londres : Routledge \& Kegan Paul.

Fadiga, L, Rizzolatti, G.,. Matelli, M., Bettinardi, V., Paulesu, E., Perani, D. et FAZIO, G. 1996, « Localization of Grasp Representations in Humans by PET: 1. Observation versus Execution », Experimental Brain Research, 111, 246-252.

FEAGIN, S. 1996 Reading with Feeling : The Aesthetics of Appreciation Ithaca : Cornell U.P.

GALLESE, V. 2005, «Intentional Attunement. The Mirror Neuron system and Its Role in Interpersonal Relations », http://www.interdisciplines.org/mirror/papers/1

Gallese, V. \& A. Goldman 1998 Mirror Neurons and the Simulation Theory of Mind Reading, Trends in Cognitive Sciences, 2, 12, 493-501.

GenetTe, G. 1972 Figures III Editions du Seuil Paris.

GODøY, R. I. 2001. «Imagined Action, Excitation, and Resonance », in R. I. GoDOY et H. JORGENSEN (eds.), Elements of Musical Imagery pp. 239-252. Lisse: Swets and 
Zeitlinger.

Godoy, R. I. \& JøRGEnSEN, H. Eds. 2001. Elements of Musical Imagery. Lisse: Swets et Zeitlinger.

Goldman, A. 1992, «Empathy, Mind, and Morals. Proceedings and Addresses of the American Philosophical Association », 66, No. 3, pp. 17-41.

Goldman, A. 2000, «The Mentalizing Folk », in D. SPERBER (ed.), Metarepresentations : a Multidisciplinary Perspective, Oxford : Oxford University Press.

Goldman, A., et SRIPADA, C.S. 2004, « Simulationist Models of Face-based Emotion Recognition », Cognition, in press.

Gombrich, E. 1965, «Meditations on a Hobby Horse, or the Roots of Artistic Form », In Meditations on a Hobby Horse and Other Essays on the Theory of Art. Londres Phaidon.

GordOn, R. 1995a, «The Simulation Theory: Objections and Misconceptions », in DAVIES \& STONE (eds.), 1995, 100-122.

Gordon, R. 1995b, «Folk Psychology as Simulation », in DAVIES \& STONE (eds.), 1995, 60-73.

GORDON, R., 1996, « Radical Simulation », in CARRUTHERS \& SMITH (eds) 1996, 1121.

Gould, S. et Lewontin, R. 1979, «The Spandrels of San Marco and the Panglossian Paradigm », Proceedings of the Royal Society, B205, 581-598.

GRÈZES, J. \& de GELDER, B. (2005). "Contagion motrice et contagion émotionnelle". In Andrès, C., BArthélémy, C., Berthoz, A., Massion, J., \& Rogé, B. (Ed.). Autisme, cerveau et développement : de la recherche à la pratique, pp. 293-318. Odile Jacob.

Hamburger, K. 1957. Die Logik der Dichtung Stuttgart : Ernst Klett Verlag. Traduction française de P. Cadiot, Logique des genres littéraires Paris : Editions du Seuil, 1986.

HARRIS, P. 1993, « Pretending and Planning », In S. BARON-COHEN, H. TAGERFLUSBERG and D.COHEN (eds.), Understanding Other Minds: Perspectives from Autism.. Oxford University Press.

HeAL, J., 1994, «Simulation vs. Theory Theory: What is the Issue? », in C. PEACOCKE (ed.), Objectivity, Simulation, and the Unity of Consciousness, Oxford: Oxford University Press, 129-144.

HEAL, J., 1996, « Simulation, Theory and Content », in CARRUTHERS \& Smith 1996 (eds.)

HEAL, J., 1998, « Co-Cognition or Off-Line Simulation: Two Ways of Understanding the Simulation Approach », Mind and Language 13:4, 477-498. 
JACOB, P. 2002 "The Scope and Limits of Mental Simulation », in Dokic \& Proust (eds.), 87-109.

JACOB P \& JEANNEROD M (2005) "The motor theory of social cognition: A critique". Trends in the Cognitive Sciences 9: pp. 21-25.

JANATA, P. 2001, « Neurophysiological Mechanisms Underlying Auditory Image Formation in Music ». In R. I. Godøy \& H. Jørgensen (eds.), Elements of Musical Imagery Lisse: Swets \& Zeitlinger Publishers.

Kahneman, D. \& A. TVERSKy 1979, «TheSimulation Heuristic », in D. Kahneman, P. Slovic \& A. Tversky (eds.)1982 Judgment under Uncertainy: Heuristics and Biases Cambridge: Cambridge University Press.

LEVINSON, J. Sound, « Gesture, Spatial Imagination and the Expression of Emotion in Music », European Review of Philosophy 5 2002: 137-150

LIPPS, T. 1903. Grundlegung der Aesthetik . Bamberg und Leipzig: W. Engelmann.

Magnée, M. J. C. M., Stekelenburg, J. J., Kemner, C., \& de Gelder, B. (2007).

"Similar facial EMG responses to faces, voices, and body expressions".

NeuroReport, 18, (4), pp. 369-372.

Meltzoff, A. et M. Moore, 1983, « Newborn Infants Imitate Adult Facial Gestures », Child Development, 54, 702-709.

METZ, C. 1977, Le Signifiant Imaginaire, coll. 10/18 , Paris : UGE.

NAdel J, DeCETy J. (eds.) 2002 Imiter pour Découvrir L'Humain : Psychologie, Neurobiologie, Robotique, Philosophie de L'Esprit. PUF Paris.

PAVEL, T. 2000, « Fiction and Imitation », Poetics Today 21:3, 521-541.

Pelletier, J. 2005 «Deux Conceptions de l'Interprétation des Récits de Fiction », Philosophiques 32/1, pp. 39-54.

Plantinga, Carl, 1999, « Scene of Empathy and the Human Face », in Passionate Views, ed. Carl Plantinga et Greg M. Smith John Hopkins University, pp. 239-255.

Rizzolatti, G., Camarda, R., Fogassi, L., Gentilucci, M., Luppino, G. \& Matelli, M. 1988 «Functional Organization of Area 6 in the Macaque Monkey. II. Area F5 and the Control of Distal Movements », Experimental Brain Research, 71: 491-507.

Rizzolatti, G., Fadiga, L., Gallese, V. et Fogassi, L. 1995 «Premotor Cortex and the Recognition of Motor Actions », Cognitive Brain Research, 3, 131-141.

Rizzolatti, G., Fogassi, L, Gallese, V. 2000, « Cortical Mechanisms Subserving Object Grasping and Action Recognition: a New View on the Cortical Motor Functions », in Gazzaniga, M.S. (ed.) The Cognitive Neurosciences (seconde édition), Cambridge, Mass.: MIT Press, pp. 539-552.

Rizzolatti G., Fogassi L., Gallese V. 2002, «Motor and Cognitive Functions of the Ventral 
Premotor Cortex ». Curr. Op. Neurobiol. 12:149-154, 2002.

RYLE, G. 1949 The Concept of Mind Chicago : University of Chicago Press

SCHAEFFER, J-M. 1999 Pourquoi la fiction?, Le Seuil, coll. "Poétique"

SCHELER, M. 1954 The Nature of Sympathy, Hamden, CN: Archon Books.

Walton, K. L. 1990 : Mimesis as Make-Believe - On the Foundations of the Representational Arts, Cambridge, Mass. : Harvard University Press

Walton, K. 1997a, «Spelunking, Simulation and Slime: On Being Moved by Fiction », In M. Hjort and S. Laver eds. Emotion and the Arts. Oxford University Press.

Walton, K. 1997b, «On Pictures and Photographs: Objections Answered », in R. Allen et M. Smith (eds.) Film Theory and Philosophy Oxford: Oxford University Press.

WAlton, K. 1999, «Projectivism, Empathy, and Musical Tension », Philosophical Topics, Vol. 26, $\mathrm{N}^{\circ} 1 \& 2,407-440$.

WiCKer, B., Keysers, C., Plailly, J., Royet, J-P., Gallese, V., and Rizzolatti, G. 2003, « Both of Us Disgusted in My Insula: The Common Neural Basis of Seeing and Feeling Disgust », Neuron, 40: 655-664. 